Migration and proliferation dichotomy in tumor cell invasion

Fedotov, Sergei and Lomin, Alexander

2007

MIMS EPrint: 2007.146

Manchester Institute for Mathematical Sciences

School of Mathematics

The University of Manchester

\footnotetext{
Reports available from: http://eprints.maths.manchester.ac.uk/

And by contacting: The MIMS Secretary

School of Mathematics

The University of Manchester

Manchester, M13 9PL, UK
} 


\title{
Migration and Proliferation Dichotomy in Tumor-Cell Invasion
}

\author{
Sergei Fedotov ${ }^{1}$ and Alexander Iomin ${ }^{2}$ \\ ${ }^{1}$ School of Mathematics, The University of Manchester, Manchester M60 1QD, United Kingdom \\ ${ }^{2}$ Department of Physics, Technion, Haifa, 32000, Israel \\ (Received 17 October 2006; published 12 March 2007)
}

\begin{abstract}
We propose a two-component reaction-transport model for the migration-proliferation dichotomy in the spreading of tumor cells. By using a continuous time random walk (CTRW), we formulate a system of the balance equations for the cancer cells of two phenotypes with random switching between cell proliferation and migration. The transport process is formulated in terms of the CTRW with an arbitrary waiting-time distribution law. Proliferation is modeled by a standard logistic growth. We apply hyperbolic scaling and Hamilton-Jacobi formalism to determine the overall rate of tumor cell invasion. In particular, we take into account both normal diffusion and anomalous transport (subdiffusion) in order to show that the standard diffusion approximation for migration leads to overestimation of the overall cancer spreading rate.
\end{abstract}

PACS numbers: 87.17.Aa, 05.40.-a, 05.40.Fb, 87.15.Vv

Extensive investigations have been devoted to the modeling of cancerous growth [1-3]. Although a great deal of progress has been made in this theory, especially for solid tumors, for which growth is basically due to cell proliferation, our understanding of malignant gliomas, the diffusive and highly invasive brain tumors, is much less complete [2]. The main reason for this is that unlike solid tumors, gliomas not only are able to proliferate but also to invade the surrounding brain parenchyma actively. The surgical resection of diffusive tumors is ineffective since the cancer cells have already invaded the surrounding brain tissue. This leads to recurrence of tumor, and the prognosis for patients suffering from malignant gliomas is very poor. Thus, proliferation and especially migration of gliomas provide a significant challenge for modeling, and this is why the invasiveness of tumors has been studied extensively in recent years $[2,4,5]$.

Invasion, itself, is a very complex process of receptormediated transport [6], which involves several steps of cell migration and proliferation (see a review [4]). Experimental evidence shows the lower proliferation rate of migratory cells in comparison with the tumor core, which indicates an inverse correlation between mobility and proliferation of cell population. The existence of this important phenomenon was supported by numerous experimental data obtained in vitro and clinical data obtained in vivo [4]. It was formulated by Giese et al. [7] as a migration-proliferation dichotomy. It turns out that proliferation and migration of tumor cells are mutually exclusive phenotypes: the spreading suppresses cell proliferation and visa versa. The molecular mechanism for this dichotomy has been suggested in [8]; and then an active implementation for the numerical modeling of the brain tumor and its fractional topology has been established [9]. It turns out that this behavior of cells is an inherent process of a socalled continuous time random walk (CTRW). This transport concept, based on jump and waiting-time distributions, has been extensively and successfully employed for numerous applications [10,11]. Migration-proliferation dichotomy was formulated in the framework of the CTRW in [12]. The primary focus was on the influence of cell fission on transport properties of cells. An essential decrease in cell motility during fission time, or their self entrapping, is determined by the interaction of cells with their environment. In vitro experimental observations of cell transport confirm the essential decrease in cell motility during cell proliferation [13].

Usually the random mobility of tumor cells is described by Fick's law. However, it has been shown that the diffusion approximation for the transport process together with a logistic growth yields an overestimation of the overall propagation rate $[14,15]$. Since the tumor cells' migration is the most critical feature of brain cancer, causing treatment failure, the transport has to be properly understood. One of the main purposes of this Letter is to take into account anomalous transport (subdiffusion) leading to slow mobility of cancer cells in the invasive zone.

Here, we propose an alternative approach for the migration-proliferation dichotomy. We employ a twocomponent CTRW, assuming that the glioma cells are of two phenotypes. In state 1 (migratory phenotype), the cells randomly move but there is no cell fission. In state 2 (proliferating phenotype), the cancer cells do not migrate and only proliferation takes place. The exact mechanism of switching between the two phenotypes is not known. An interesting deterministic mechanism for this phenotype switch has been suggested recently in [16]. However, the mathematical modeling involves many parameters, some of which are difficult to estimate. We propose the stochastic approach for the proliferation-migration switching involving only two parameters. We assume that the cell of type 1 remains in a state 1 during a waiting time $\tau_{1}$ and then switches to a cell of type 2. After a waiting time $\tau_{2}$, spent in a state 2 , it switches back to a cell of type 1 . Both waiting times $\tau_{1}$ and $\tau_{2}$ are mutually independent random variables. In this Letter, we consider them exponentially 
distributed with parameters $\beta_{1}$ and $\beta_{2}$ :, namely, $f\left(\tau_{i}\right)=$ $\beta_{i} \exp \left(-\beta_{i} \tau_{i}\right)$. Here the parameters $\beta_{i}$ are the switching rates, namely, $\beta_{1}$ is the switching rate from state 1 to 2 , while $\beta_{2}$ determines the transition rate $2 \rightarrow 1$. The last parameter can control the lower migratory cell proliferation.

Let us introduce the density for the cells of migratory phenotype (cells of type 1), $n_{1}(t, x)$, and for the cells of proliferating phenotype (cells of type 2$), n_{2}(t, x)$. The balance equations can be written as follows:

$$
\begin{aligned}
& n_{1}(t, x)=n_{1}(0, x) \Psi(t) e^{-\beta_{1} t}+\int_{0}^{t} \int n_{1}(t-s, x-z) \rho(z) \\
& \times \psi(s) e^{-\beta_{1} s} d z d s+\beta_{2} \int_{0}^{t} n_{2}(t-s, x) \Psi(s) \\
& \times e^{-\beta_{1} s} d s \\
& n_{2}(t, x)= \\
& \quad n_{2}(0, x) e^{-\beta_{2} t}+U \int_{0}^{t} n_{2}(t-s, x) \\
& \quad \times\left[1-n_{2}(t-s, x) / K\right] e^{-\beta_{2} s} d s \\
& \quad+\beta_{1} \int_{0}^{t} n_{1}(t-s, x) e^{-\beta_{2} s} d s
\end{aligned}
$$

where $\rho(z)$ is the probability density for migration jump length, while $\psi(s)$ is the probability density of waiting times between jumps, and $\Psi(t)=1-\int_{0}^{t} \psi(s) d s$ is the probability that a cell of type 1 makes no jump until time $t$. The exponential factor $e^{-\beta_{i} t}=\int_{t}^{\infty} f\left(\tau_{i}\right) d \tau_{i}$ is the probability that cells of phenotype $i$ do not switch until time $t$. Equation (1) describes the balance of cells of type 1 at time $t$ at position $x$. The first term on the right hand side of the equation represents those cells of type 1 that stay up to time $t$ at position $x$ such that no jump occurs, and no switch $1 \rightarrow$ 2 takes place. The independence of the random jumps and switching gives us the probability $\Psi(t) e^{-\beta_{1} t}$ while the first factor $n_{1}(0, x)$ is the initial density of cells of type 1 . The second term describes the number of cells of type 1 arriving at $x$ up to time $t$ due to the following random mechanism of migration: the cell of type 1 at time $t-s$ at position $x-z$ waits a random time $s$ before jumping at a distance $z$ and remains a cell of type 1 . This process is determined by the transition probability $\psi(s) \rho(z)$. The limits of the space integral are determined by the boundaries. The last term in Eq. (1) represents the number of cells of type 2 that switches to the cell of type 1 up to time $t$ and remaining the cells of type 1 (due to the factor $e^{-\beta_{1} s}$ ). It also takes into account the fact that if transition $2 \rightarrow 1$ happens at time $t-s$, then no jump takes place during the remaining time $s$ [due to the factor $\Psi(s)$ ].

Regarding Eq. (2), the first term on the right hand side has the same physical meaning as one in Eq. (1). The second term is the logistic growth [17] for cells of type 2 , which occurs providing that no switch takes place up to time $t$. Here, $U$ is the cell proliferation rate, and $K$ is the carrying capacity of the environment. The last term of
Eq. (2) represents the number of cells of type 1 switching to the state 2 over the time interval $(0, t)$. Note that onecomponent balance equation involving transport and production term has been analyzed in [15].

The balance Eqs. (1) and (2) can be written as the system of integro-differential equations. By using the Laplace transform $\tilde{n}_{i}(H)=\int_{0}^{\infty} e^{-H t} n_{i}(t) d t$ and presenting the left hand side of the equations in the form $H \tilde{n}_{i}(H)-\tilde{n}_{i}(0)$ which is the Laplace transform of the time derivative, one obtains

$$
\begin{gathered}
\frac{\partial n_{1}}{\partial t}=\int_{0}^{t} \alpha(t-s) \int\left[n_{1}(s, x-z)-n_{1}(s, x)\right] \rho(z) d z d s \\
-\beta_{1} n_{1}+\beta_{2} n_{2}, \\
\frac{\partial n_{2}}{\partial t}=U n_{2}\left(1-n_{2} / K\right)+\beta_{1} n_{1}-\beta_{2} n_{2},
\end{gathered}
$$

where the "memory" kernel $\alpha(t)$ is defined in terms of its Laplace transform

$$
\tilde{\alpha}(H)=\frac{\left(H+\beta_{1}\right) \tilde{\psi}\left(H+\beta_{1}\right)}{\left[1-\tilde{\psi}\left(H+\beta_{1}\right)\right]}
$$

with $\tilde{\psi}(H)=\int_{0}^{\infty} \psi(t) e^{-H t} d t$. The equivalence of onecomponent balance equation to a master equation involving memory kernel has been shown in [10]. Note that it is impossible to find an explicit expression for memory kernel $\alpha(t)$ for arbitrary choices of waiting-time pdf $\psi(t)$. In what follows, we will be concerned with the overall rate of the spreading of gliomas. It turns out that this rate depends on the Laplace transform $\tilde{\alpha}(H)$ rather than $\alpha(t)$. That is why the formula (5) plays a crucial role in this Letter.

It follows from observations [4] that cell jumps are controlled by receptor-mediated adhesion of tumor cells to matrix proteins, and jump lengths are very small. Therefore, $\rho(z)$ in Eq. (3) is a rapidly decaying function for large $z$, and the long-range effects are weak. In this case, one can use the Taylor series in Eq. (3) expanding $n_{1}(s, x-z)$ in $z$ and truncate the series at the 2 nd moment. It should be stressed that this truncation is a well-defined procedure since the higher moments for such a kernel become progressively smaller [18]. Assuming the spatial symmetry of $\rho(z)$ (there is no convection: $\int z \rho(z) d z=0$ ), we obtain from Eq. (3) [19]

$$
\frac{\partial n_{1}}{\partial t}=\frac{\sigma^{2}}{2} \int_{0}^{t} \alpha(t-s) \frac{\partial^{2} n_{1}}{\partial x^{2}} d s-\beta_{1} n_{1}+\beta_{2} n_{2},
$$

where $\sigma^{2}=\int z^{2} \rho(z) d z$. Generalization on 3D is straightforward; namely, the second derivative is replaced by the Laplace operator $\Delta$.

Now we are in a position to find the overall rate $u$ at which a wave front of the cancer cells spreads. In the classical setting based on Fisher equation [17], the propa- 
gation rate $u$ is proportional to $\sqrt{D U}$, where $D$ is the diffusion coefficient and $U$ is the proliferation rate. In this Letter, we have a couple of Eqs. (4) and (6), and we are interested in a traveling wave solution to this system and its propagation rate $u$. If we specify frontlike initial conditions for densities $n_{1}$ and $n_{2}$, the fronts for both densities quickly achieve the stationary forms that propagate with a constant rate $u$. The main purpose here is to find the dependence of this propagation rate on the statistical characteristics of the random switching process, $\beta_{1}$ and $\beta_{2}$, and random walk in space, $\sigma^{2}$ and $\psi(t)$. The objective here is to find the rate $u$ without resolving the shape of the traveling waves $[14,20]$. For this purpose, we use a hyperbolic scaling $x \rightarrow x / \varepsilon, t \rightarrow t / \varepsilon$ and the rescaled densities $n_{i}^{\varepsilon}(t, x)=n_{i}(t / \varepsilon, x / \varepsilon)$. We apply the exponential transformation

$$
n_{i}^{\varepsilon}(t, x)=A_{i} \exp \left(-\frac{G(t, x)}{\varepsilon}\right), \quad i=1,2
$$

in Eqs. (4) and (6), where positive constant $A_{1}$ and $A_{2}$ represent the stable equilibrium points of the densities $n_{1}^{\varepsilon}$ and $n_{2}^{\varepsilon}$. Our purpose is to find an equation for $G(t, x)$ which gives us the spreading front position $x(t)$ in the limit of the long time and large distance, from the equation $G[t, x(t)]=0$ [14]. To ensure the minimal spreading rate, we use the frontlike initial conditions: $n_{i}(0, x)=A_{i}$ for $x<0$ and $n_{i}(0, x)=0$ for $x \geq 0$ [20]. Substituting (7) into the equations for $n_{1}^{\varepsilon}, n_{2}^{\varepsilon}$ that are derived from Eqs. (4) and (6), one obtains two equations for $A_{1}$ and $A_{2}$ in the limit $\varepsilon \rightarrow 0$. This system has a nontrivial solution when the corresponding determinant is equal to zero. This yields a generalized Hamilton-Jacobi equation, involving the first two derivatives $\partial G / \partial t$ and $\partial G / \partial x$ :

$$
\begin{aligned}
&\{1\left.-\left[1+\frac{\sigma^{2}}{2}\left(\frac{\partial G}{\partial x}\right)^{2}\right] \int_{0}^{\infty} e^{(\partial G / \partial t) s} \psi(s) e^{-\beta_{1} s} d s\right\} \\
& \times\left[1-U \int_{0}^{\infty} e^{(\partial G / \partial t) s} e^{-\beta_{2} s} d s\right] \\
&-\beta_{1} \beta_{2} \int_{0}^{\infty} e^{(\partial G / \partial t) s} \Psi(s) e^{-\beta_{1} s} d s \int_{0}^{\infty} e^{(\partial G / \partial t) s} e^{-\beta_{2} s} d s \\
&=0 .
\end{aligned}
$$

Note that inferring Eq. (8), we do not make any assumptions regarding waiting-time pdf $\psi(t)$. If we introduce the Hamiltonian function $H=-\partial G / \partial t$, the generalized momentum $p=\partial G / \partial x$, and the Laplace transform $\tilde{\psi}(H)=$ $\int_{0}^{\infty} \psi(t) e^{-H t} d t$, then the Hamilton-Jacobi Eq. (8) takes the form

$$
\frac{\sigma^{2} p^{2}}{2}=\frac{1}{\tilde{\psi}\left(H+\beta_{1}\right)}\left[1-\frac{\beta_{1} \beta_{2}\left[1-\tilde{\psi}\left(H+\beta_{1}\right)\right]}{\left(H+\beta_{1}\right)\left(H+\beta_{2}-U\right)}\right]-1 .
$$

The latter equation is important since it allows us to find the overall spreading rate $u=\min _{H}\{H / p(H)\}$ by using
[14]

$$
u=\frac{H}{p(H)}, \quad \frac{\partial p}{\partial H}=\frac{p(H)}{H} .
$$

In the symmetrical 3D case, Eq. (9) corresponds to the Hamiltonian motion in the radial direction. Let us illustrate the use of the above theory through two typical distributions for the waiting-time pdf $\psi(t)$.

First, we consider a probability distribution function for the exponentially distributed waiting times: $\psi(t)=$ $\tau^{-1} e^{-t / \tau}$. We find $\tilde{\psi}(H)=(1+H \tau)^{-1}$ and $\tilde{\alpha}(H)=\tau^{-1}$, and therefore $\alpha(t)=\tau^{-1} \delta(t)$. This corresponds to the classical Fick's law for transport with the diffusion coefficient $D=\sigma^{2} / 2 \tau$. Thus, we have a classical system of reaction-diffusion equations such that the equation for the migratory cells is

$$
\frac{\partial n_{1}}{\partial t}=D \frac{\partial^{2} n_{1}}{\partial x^{2}}-\beta_{1} n_{1}+\beta_{2} n_{2} .
$$

The momentum $p(H)$ can be found from (9)

$$
p^{2}=\frac{\left(H+\beta_{1}\right)}{D}-\frac{\beta_{1} \beta_{2}}{D\left(H+\beta_{2}-U\right)} .
$$

If we assume that $\beta_{1}=\beta_{2}$, we can find from (10) and (12) $p=(U / D)^{1 / 2}$, and $H=U$. Therefore, the spreading rate is $u_{0}=(U D)^{1 / 2}$ which is half of the classical Fisher-KPP propagation speed. This is a very interesting result showing that the propagation rate is independent of the random migration-proliferation switching when cell transport is the Brownian motion and $\beta_{1}=\beta_{2}$. When $\beta_{1} \neq \beta_{2}$, one can find the ratio of the propagation rate $u$ and $u_{0}=$ $(U D)^{1 / 2}$ as

$$
\left(\frac{u}{u_{0}}\right)^{2}=\frac{H^{2}\left(H+\beta_{2}-U\right)}{U\left[\left(H+\beta_{2}-U\right)\left(H+\beta_{1}\right)-\beta_{1} \beta_{2}\right]} .
$$

The situation changes for the power law distribution (anomalous transport): $\psi(t) \sim(\tau / t)^{1+\gamma}$ with $0<\gamma<1$. This is the case when the mean waiting-time is divergent: $\langle t\rangle=\infty$. This assumption alone leads to the temporal fractional differential operator and corresponding anomalous diffusion equation [11]. The mean squared displacement for mobile cells is

$$
\left\langle x^{2}(t)\right\rangle=\frac{4 D_{\gamma}}{\Gamma(1+\gamma)} t^{\gamma},
$$

where $D_{\gamma}=\sigma^{2} / 2 \tau^{\gamma}$ is the generalized diffusion coefficient with the dimension $\mathrm{cm}^{2} \mathrm{~s}^{-\gamma}$. One of the main aims of this Letter is to find the overall propagation of cancer cells as a result of interaction of the anomalous migration (14), logistic proliferation, and random migration-proliferation switching. For this purpose, it is more convenient to define $\psi(t)$ by its Laplace transform $\tilde{\psi}(H)=\left[1+(H \tau)^{\gamma}\right]^{-1}[11]$, such that the momentum $p(H)$ can be found from (12) 


$$
p^{2}=\frac{\left(H+\beta_{1}\right)^{\gamma}}{D_{\gamma}}-\frac{\beta_{1} \beta_{2}\left(H+\beta_{1}\right)^{\gamma-1}}{D_{\gamma}\left(H+\beta_{2}-U\right)} .
$$

This formula together with (10) allows us to find the overall propagation rate of tumor cells $u_{\gamma}$ in the fractional diffusion case. It is clear that the case $\gamma=1$ corresponds to the normal diffusion approximation for cell migration (see (12)). One can find from (10), (12), and (15) the ratio of the anomalous propagation rate $u_{\gamma}$ and the normal rate $u$ determined by (13)

$$
\frac{u_{\gamma}}{u}=\left(H_{\gamma} \tau+\beta_{1} \tau\right)^{1-\gamma / 2},
$$

where $H_{\gamma}$ is the solution of $\partial p / \partial H=p(H) / H$. Since the "microscopic" time $\tau$ is much smaller than the characteristic "cell proliferation" time $U^{-1}$ and switching time $\beta_{1}^{-1}$ and $H_{\gamma} \sim U$, we conclude that $H \tau+\beta_{1} \tau<1$. It follows from (16) that the ratio $u_{\gamma} / u$ increases with $\gamma$ in the interval $0<\gamma<1$. This means that the standard diffusion approximation leads to overestimation of the overall cancer spreading. It is clear from these two examples of normal and anomalous diffusions that the advantage of balance Eqs. (1) and (2) is that they are related to "mesoscopic" description of migratory cancer cells, and give us the statistical meaning of the reaction-diffusion equations or fractional equations that are introduced usually phenomenologically.

In summary, we present a two-component model for a migration-proliferation dichotomy in the spreading of tumor cells in the invasive zone. We use a probabilistic approach based on the CTRW theory for migration, logistic growth, and random proliferation-migration switching with exponentially distributed waiting times. Our approach is not restricted to the specific mechanism of proliferation described by a logistic growth. Moreover, Eq. (2) for proliferation can be accompanied by a nutrient control or chemotaxis [21]. The main point of the Letter is that cancer cell transport is subdiffusive rather than diffusive described by Fick's law. The advantage of our approach is that it allows us to take into account anomalous (subdiffusive) transport within the general scheme of migration, proliferation, and phenotype switching. We show the equivalence of balance equations to a system of master equations involving memory kernels for the transport of mobile cells. By using a hyperbolic scaling and Hamilton-Jacobi formalism, we derive formulae for the overall spreading rate of cancer cells. We show that the memory effects (subdiffusion) lead to a decrease in propagation rate compared to a standard diffusion approximation for transport.

This research was supported by EPSRC Grant No. EP/ D03115X/1. A. I. was supported by the Israel Science Foundation.
[1] N. Bellomo and L. Preziosi, Mathematical and Computer Modelling 32, 413 (2000).

[2] K. Swanson et al., J. Neurol. Sci. 216, 1 (2003).

[3] Mathematical Modeling of Biological Systems, edited by A. Deutsch, L. Brusch, H. Byrne, G. de Vries, and H.-P. Herzel, Biomedical Applications Vol. I (Birkhäuser, Boston, 2007)

[4] A. Giese et al., J. Clin. Oncol. 21, 1624 (2003).

[5] L. M. Sander and Th.S. Deisboeck, Phys. Rev. E 66, 051901 (2002); S. Habib, C. Molina-Paris, and Th. S. Deisboeck, Physica A (Amsterdam) 327, 501 (2003).

[6] T. Bollenbach, K. Kruse, P. Pantazis, M. Gonzáles-Gaitán, and F. Jülicher, Phys. Rev. Lett. 94, 018103 (2005).

[7] A. Giese et al., International Journal of cancer Journal international du cancer 67, 275 (1996).

[8] A. Wells, Int. J. Biochem. Cell Biol. 31, 637 (1999).

[9] Y. Mansury and T. Deisboeck, Physica A (Amsterdam) 331, 219 (2004); Physica D (Amsterdam) 196, 193 (2004).

[10] E. W. Montroll and B. J. West, in Fluctuation Phenomena, edited by E.W. Montroll and J.L. Lebowitz (NorthHolland, Amsterdam, 1979).

[11] R. Metzler and J. Klafter, Phys. Rep. 339, 1 (2000).

[12] A. Iomin, Phys. Rev. E 73, 061918 (2006); J. Phys.: Conf. Ser. 7, 57 (2005); The World Scientific and Engineering Academy and Society Trans. Biol. Biomed. 2, 82 (2005).

[13] P. Dieterich (private communication).

[14] S. Fedotov, Phys. Rev. Lett. 86, 926 (2001).

[15] S. Fedotov and V. Méndez, Phys. Rev. E66, 030102 (2002); S. Fedotov and Y. Okuda, Phys. Rev. E 66, 021113 (2002).

[16] C. A. Athale, Y. Mansury, and T. S. Deisboeck, J. Theor. Biol. 233, 469 (2005).

[17] J.D. Murray, Mathematical Biology (Springer-Verlag, Berlin, 1989).

[18] See an explanation of nonlocal effects in Ch. 11.5, of Ref. [17], Vol. 1.

[19] The integration limits in Eq. (5) should be independent of $\rho(z)$ and taken on $\pm \infty$. These limits satisfy, e.g., both a kernel with the compact support, where $\rho(z)=0$ for $|z|>$ $x_{0}$ and the Gaussian kernel $\rho(z)=\frac{1}{\sigma \sqrt{\pi}} \exp \left(-z^{2} / \sigma^{2}\right)$. In particular, for the Gaussian kernel, its Fourier transform is $\hat{\rho}(k)=\hat{F}[\rho(z)]=\exp \left(-k^{2} \sigma^{2}\right)$. We assume here that the characteristic length scale for macroscopic cell transport is much larger than the variance of individual jump lengths of a cell. This means that $k \sigma \ll 1$, and $\hat{\rho}(k) \approx$ $\left(1-k^{2} \sigma^{2} / 2\right)$ (see, e.g., [11]). Then, applying the Fourier transform to $\hat{F}\left[n_{1}(x)\right]=\hat{n}_{1}(k)$ and using this physical argument, we obtain that the convolution term in Eq. (5) becomes the product $\hat{n}_{1}(k)[\hat{\rho}(k)-\hat{\rho}(0)] \approx$ $k^{2} \sigma^{2} \hat{n}_{1}(k)$. Finally, performing the Fourier inversion, one obtains Eq. (8).

[20] M. Freidlin, Markov Processes and Differential Equations: Asymptotic Problems (Birkhauser, Basel, 1996).

[21] E. Khain, L. D. Sander, and A. M. Stein, Complexity 11, 53 (2005); E. Khain and L. D. Sander, Phys. Rev. Lett. 96, 188103 (2006). 